\title{
Growth mechanism of nanowires: Binary and ternary chalcogenides
}

\author{
N. B. Singh, S.R. Coriell, Ching Hua Su*, R. H. Hopkins**, B. Arnold, Fow-Sen Choa and \\ Brian Cullum \\ University of Maryland Baltimore County, 1000 Hilltop Circle, Baltimore, MD 21250 \\ * EM31 NASA/Marshall Space Flight Center, Huntsville, AL 35812 \\ **Hopkins Inc. Export, PA 15632
}

\begin{abstract}
Semiconductor nanowires exhibit very exciting optical and electrical properties including high transparency and a several order of magnitude better photocurrent than thin film and bulk materials. We present here the mechanism of nanowire growth from the melt-liquid-vapor medium. We describe preliminary results of binary and ternary selenide materials in light of recent theories. Experiments were performed with lead selenide and thallium arsenic selenide systems which are multifunctional material and have been used for detectors, acousto-optical, nonlinear and radiation detection applications. We observed that small units of nanocubes and elongated nanoparticles arrange and rearrange at moderate melt undercooling to form the building block of a nanowire. Since we avoided the catalyst, we observed self-nucleation and uncontrolled growth of wires from different places. Growth of lead selenide nanowires was performed by physical vapor transport method and thallium arsenic selenide nanowire by vapor-liquid-solid (VLS) method. In some cases very long wires (>mm) are formed. To achieve this goal experiments were performed to create situation where nanowires grew on the surface of solid thallium arsenic selenide itself.
\end{abstract}

Keywords: Nanowire, melt, chalcogenides, growth, thallium arsenic selenide, lead selenide, morphology

\section{INTRODUCTION}

In the past two decades significant investment has been made and expectation for the applications of nanotechnology is continuously increasing in almost every aspect of life. . Almost every area of research has projected improvements in sensors, or even a promise for the emergence of some novel device technologies. For these applications major focuses of research are in the areas of nanoparticles, carbon nanotubes and graphene. Some near term applications of nanowires have produced very inspiring results for photodetectors and other low light detectors. In spite of these early success based on nanowires, progress on the growth mechanism and fabrication of nanowire-based devices is very limited. Some of these studies [15] have gained momentum for short wave infrared (SWIR) avalanche photodiodes. We chose lead selenide and thallium arsenic selenide materials which can be used for devices with significant advantages over traditional night vision equipment, which generally amplifies radiation over the visible and near infrared range out to a wavelength of $1 \mu \mathrm{m}$. In this article we present the mechanism of nanowire growth and preliminary results observed with lead selenide $(\mathrm{PbSe})$ and thallium arsenic selenide $\left(\mathrm{Tl}_{3} \mathrm{AsSe}_{3}\right)$ material systems.

Keywords: Nanowire, Lead selenide, Thallium Arsenic Selenide, melt growth, Physical Vapor Deposition, Melt

\section{EXPERIMENTAL METHODS AND RESULTS}


An overview on the mechanism and growth of nanowires for variety of devices is described in references 1 and 2 . Parker et al [3] had proposed the classical model of whisker growth almost a half century ago. It is based on impingement of atoms on the whisker sides, and diffusion of these adatoms to the whisker tip where they are incorporated in the growing whisker. The vapor-liquid-solid (VLS) method used a liquid drop at the whisker tip which was an effective method of capturing atoms from the vapor. Mechanism of nanowire growth has been described recently by several authors [4-7]. However designing an experiment for nanowires it is clearly difficult especially to measure $\mathrm{L}(\mathrm{t})$ during a growth experiment. Where $\mathrm{L}(\mathrm{t})$ is length at time $\mathrm{t}$. Some attempts have been made to measure length and diameter by carrying out an experiment for a fixed time and after stopping the growth process nanowires. All these models suggest the growth which includes impingement of atoms on the substrate on the nanowire sides and on the liquid drop. The atoms on the substrate diffuse to the whisker base and then along the whisker sides to the liquid drop where they are incorporated into the nanowire. To evaluate these concepts we performed growth by vapor transport as well as melt growth. Most of the models involve three impingement rates (substrate, nanowire sides, and liquid droplet) and two surface diffusion lengths (substrate and nanowires sides). If one assumes that all the impingement rates are approximately equal, and that the surfaces diffusion lengths are the same, solving models is easy since it greatly reduces the number of parameters. The model is such that one can always pick an impingement rate to obtain the measured nanowire length. Some of the models take into account diffusion through the liquid drop on the surface of the liquid drop) and also account the interface kinetics. The simplest model to compare with experimental data is by Johansson et al [7], which describes the growth velocity of nanowires by the equation:

$$
\begin{gathered}
\frac{1}{2 \Omega} \frac{d L}{d t}=\frac{R_{w} \lambda_{w}}{r_{w}} \tanh \left(L / \lambda_{w}\right)-\frac{J_{s w}}{r_{w} \cosh \left(L / \lambda_{w}\right)}+R_{t o p} \\
J_{s w}=-R_{s} \lambda_{s} \frac{K_{1}\left(r_{w} / \lambda_{s}\right)}{K_{0}\left(r_{w} / \lambda_{s}\right)}
\end{gathered}
$$

where $\Omega$ is the atomic or molecular volume, $\mathrm{L}$ is the length of the nanowire, $\mathrm{t}$ is time, $R_{w}$ is the deposition rate on the wire sides, $\lambda_{w}$ is the diffusion length on the wire sides, $r_{w}$ is the radius of the wire, $\lambda_{s}$ is the diffusion length on the substrate, $K_{0}$ and $K_{1}$ are modified Bessel functions [3,4,7], $R_{s}$ is the deposition rate on the substrate, and $R_{t o p}$ is the deposition rate on the top of the wire.

2.1 Source materials and purification: As supplied lead selenide was listed for $99.999 \%$ purity. This material was used without any further purification. Thallium arsenic selenide $\left(\mathrm{Tl}_{3} \mathrm{AsSe}_{3}\right)$ material was synthesized from the as supplied thallium (99.999\%), Arsenic (99.9999\%) and selenium (99.9999\%). These elements were further purified before reacting. Thallium was purified by etching with water-nitric acid mixture, selenium was directionally solidified and arsenic was annealed in a sealed tube. Synthesis of stoichiometric composition $\mathrm{Tl}_{3} \mathrm{ASSe}_{3}$ was performed by sealing source material in well cleaned quartz tube and reacting at a temperature above 350C.

2.2 Growth of nanowires: For the nanowires of lead selenide we used physical vapor transport method in DENTON evaporator. The vapor pressure was high enough to achieve pressure $>100$ torr by raising temperature of the source material. In this case we performed experiments with and without seeds. Argon was used as a purge gas and also as a means to control the overall furnace pressure. NW growth was studied through a wide range of Argon partial pressures. The details of the evaporation and parameters are described in reference 2. As shown in Figure 1, when we used silicon substrates and maintained substrate temperature above $350 \mathrm{C}, \mathrm{PbSe}$ (with small residual impurities) nucleated on the substrate as cubes. In order to understand the mechanism, we stopped the growth and results shown in Figure $1(\mathrm{a} 0$ and (b) show the impingement on substrate and aligning of nanocubes. The continuous growth suggested that cubes merged together and grew as building blocks for the nanowires. For understanding the mechanism of nanowire growth, we used Si substrates and deposition for a period of 10 to 15 minutes. As the growth proceeds, $\mathrm{PbSe}$ vapor connects each cubes to make wires. The size ranges from 50$100 \mathrm{~nm}$. But as shown in Figure 3, in the case of glass substrates we observed different shapes also.

In order to demonstrate mechanism of growth we performed experiments to grow wires between two catalysts. Figure 1 (c) shows vapor grown selenide wire where we had used silicon wafer as substrate. In this case growth was controlled by using argon gas to control the flow of nutrient on the wafer. The pressure was kept very low (in 
the range of 10 torr) to avoid dense growth of wires. Because of catalyst, the shape of nucleus was not completely nanotube and diameter was slightly nonsymmetrical.

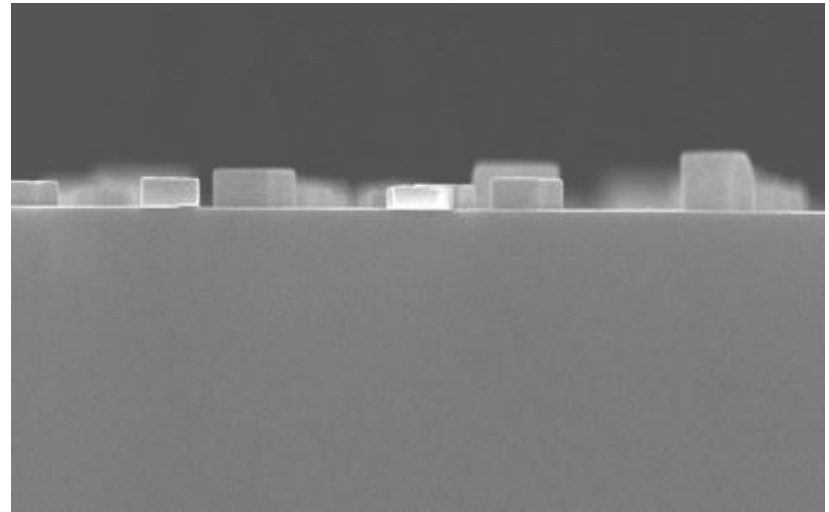

(a)

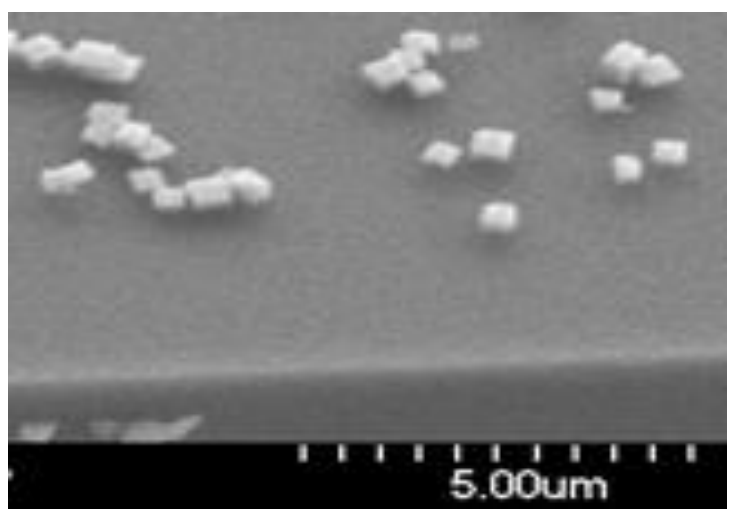

(b)

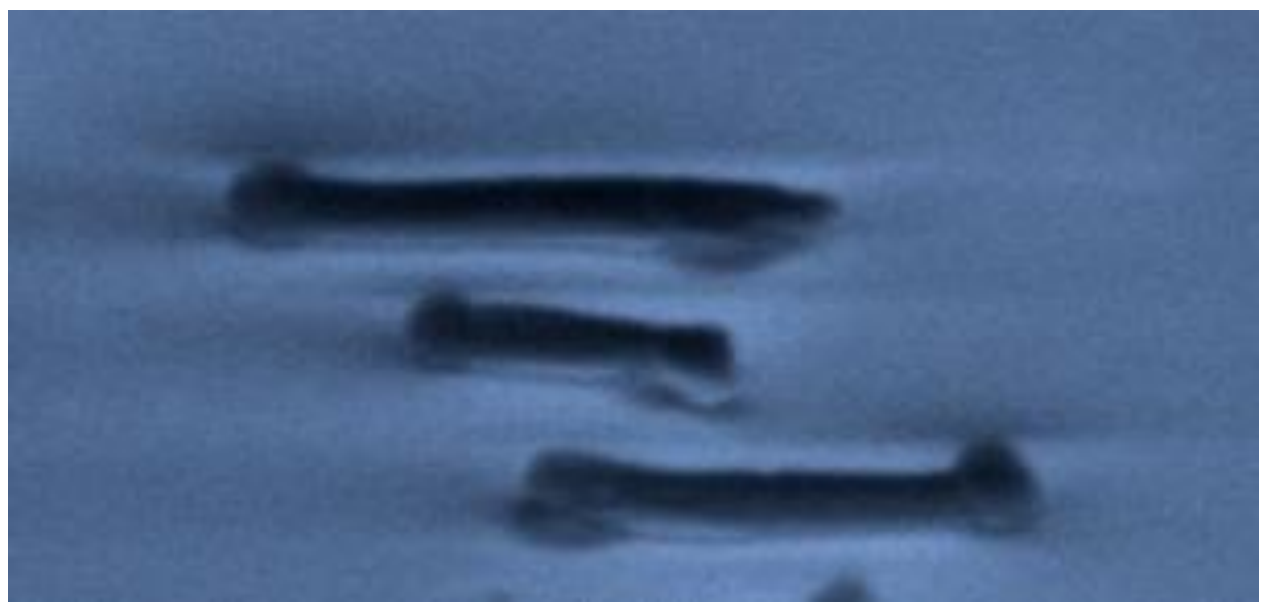

Figure 1. (a) Cubes nucleated on silicon substrates at high temperature (b) cubes aligned together as a building blocks for nanometer during PVD and (c) large diameter (200nm) selenide wire 


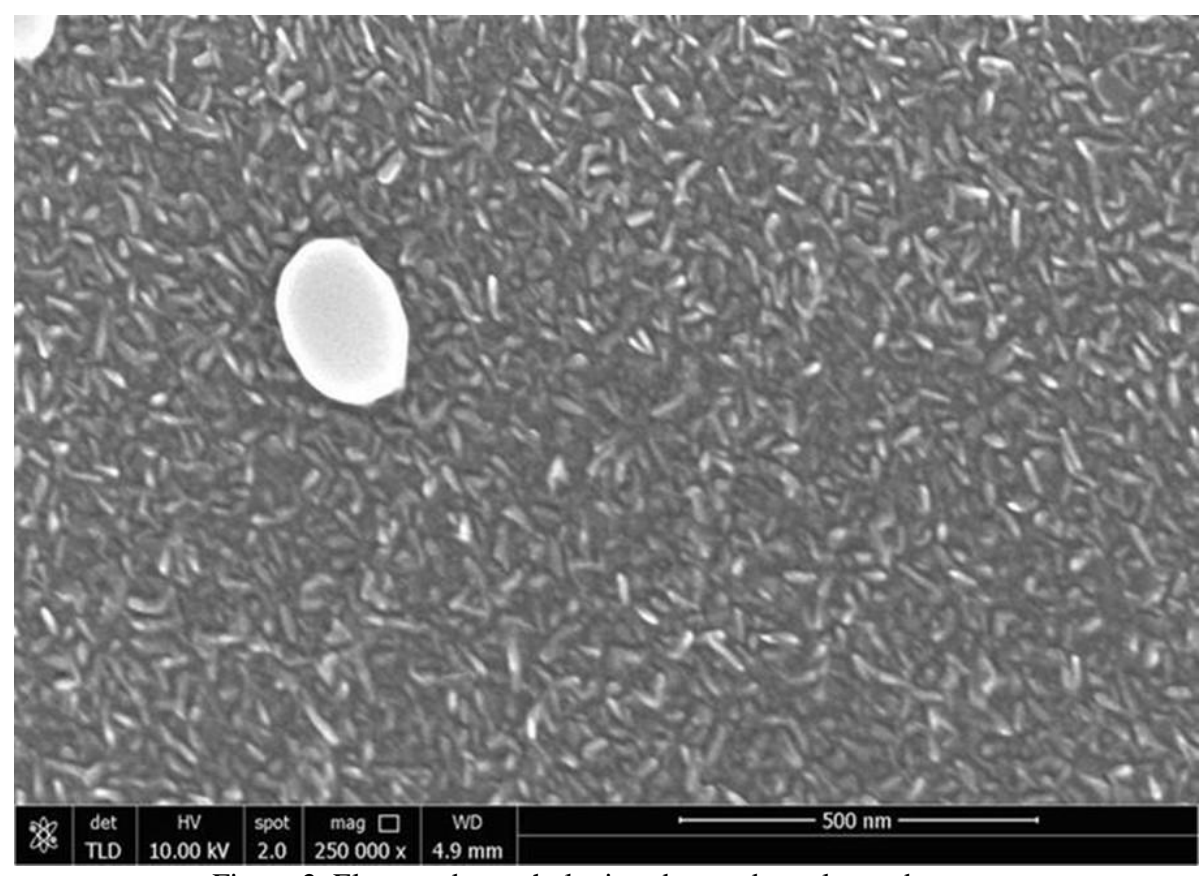

Figure 2. Elongated morphologies observed on glass substrates

Growth of lead selenide on quartz surface demonstrated very different morphology. Figure 2 shows that grains are elongated and demonstrate fat needle morphology. This indicates that substrate surface, difference in lattice parameters affects the nucleation size and morphology significantly. Although size of the catalyst has been tried to control diameter, we experimented to start self-nucleation and its effect on the size and morphology. Growth on quartz surface indicated clearly that aspect ratio and sizes were very different. Also, on quartz surface particles were in multiple crystallographic orientation and did not show any sign of merging to form nanowires.

These two experiments suggested that morphology depends on the substrate surface and lattice parameters also. For the case of thallium arsenic selenide we used the horizontal two zone furnace shown in Figure 3. The source material was placed in a $15 \mathrm{~mm}$ diameter ampoule and evacuated to drive away any residual gases. The sealed material was melted and then frozen slowly by lowering the furnace temperature. The melt-freeze cycle was repeated few times to completely mix the constituting molten components. For the growth experiments we melted the material in the horizontal quartz tube and kept the melt for 4-5 hours. After ensuring the stabilization of the temperature and melt composition, we lowered the temperature to $300-310 \mathrm{C}$, a temperature range just below the melting point $(311 \mathrm{C})$ of the $\mathrm{Tl}_{3} \mathrm{AsSe}_{3}$ composition. This provides condition for the thallium arsenic selenide nanowires growth by vapor-liquid-solid (VLS) mechanism. After few minutes material started nucleating as nanocubes on the liquid-solid surface. We observed that a very thin layer of material solidified on the surface as the cubical morphology nucleated. As the solidification proceeded, array of nano cubes were observed. Since this material was not transparent in visible region, it was not possible to observe flow of liquid during the growth. After cooling to room temperature when we studied the morphology (Figure 4), an array of nanocubes were observed. To understand the joining of these nanocubes to form nanowires, we used fast cooling. In this experiment as soon as nucleation started near melting point, we switched off the power of the furnace. The furnace cooled at the rate of $10 \mathrm{~K} / \mathrm{h}$ between melting temperature and $170 \mathrm{C}$. Below this temperature cooling rate was higher than $10 \mathrm{~K} / \mathrm{h}$ and varied significantly since we did not control the cooling. 


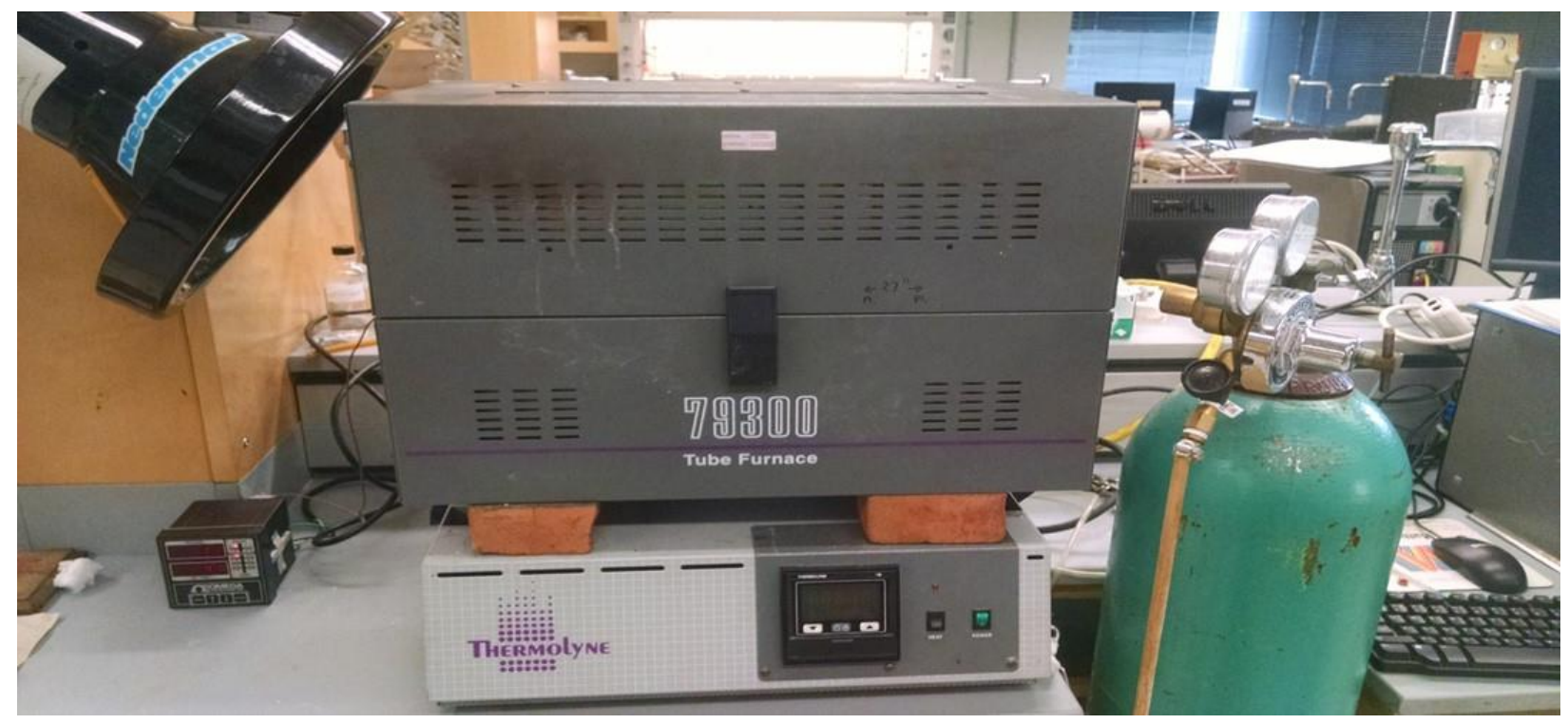

Figure 3. Horizontal furnace used for $\mathrm{Tl}_{3} \mathrm{AsSe}_{3}$ nanowire growth. This furnace suitable for melt and vapor transport growth of selenide materials

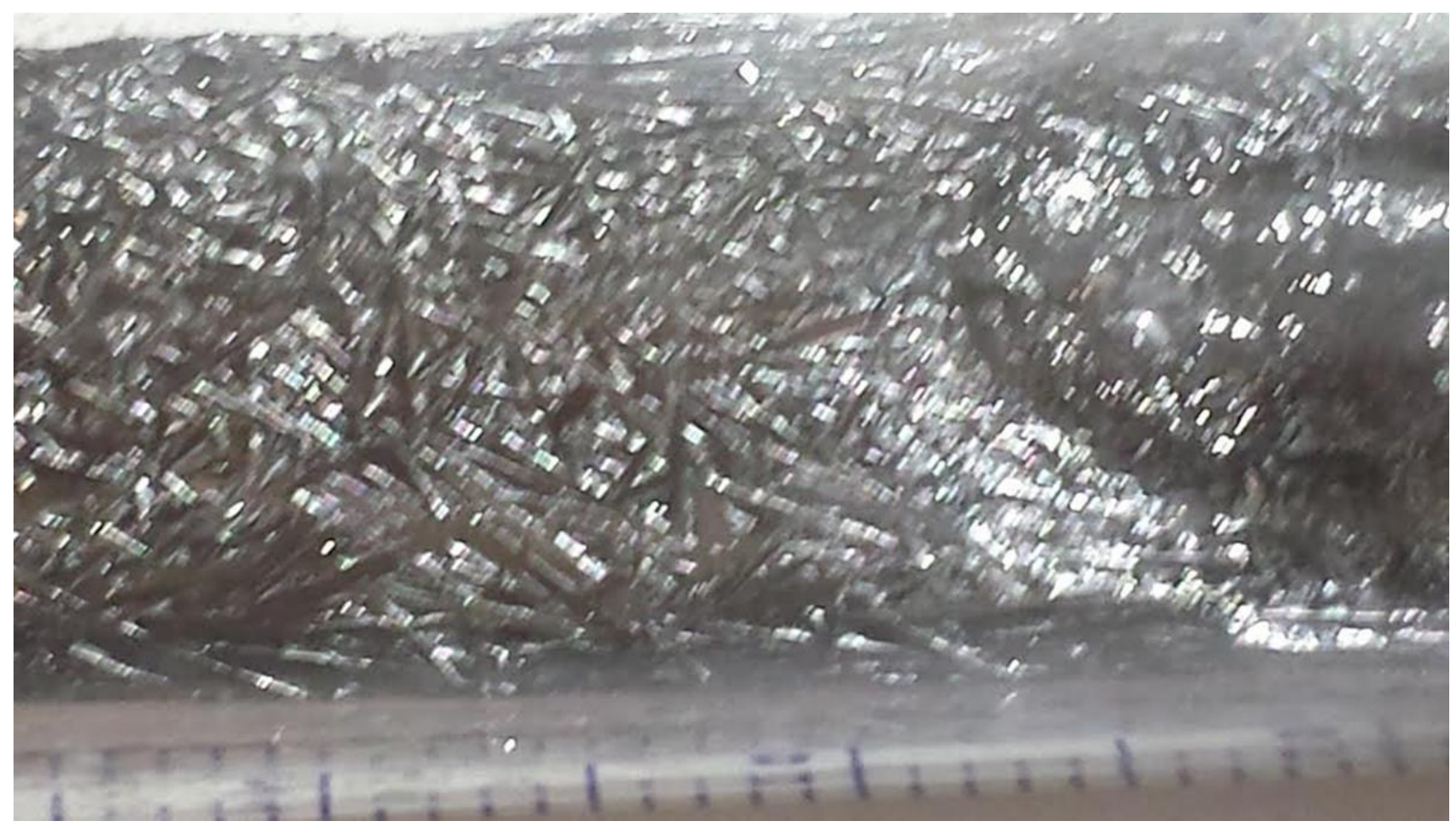

Figure 4. Nanocubes of $\mathrm{Tl}_{3} \mathrm{AsSe}_{3}$ compounds nucleated by VLS mechanism. 


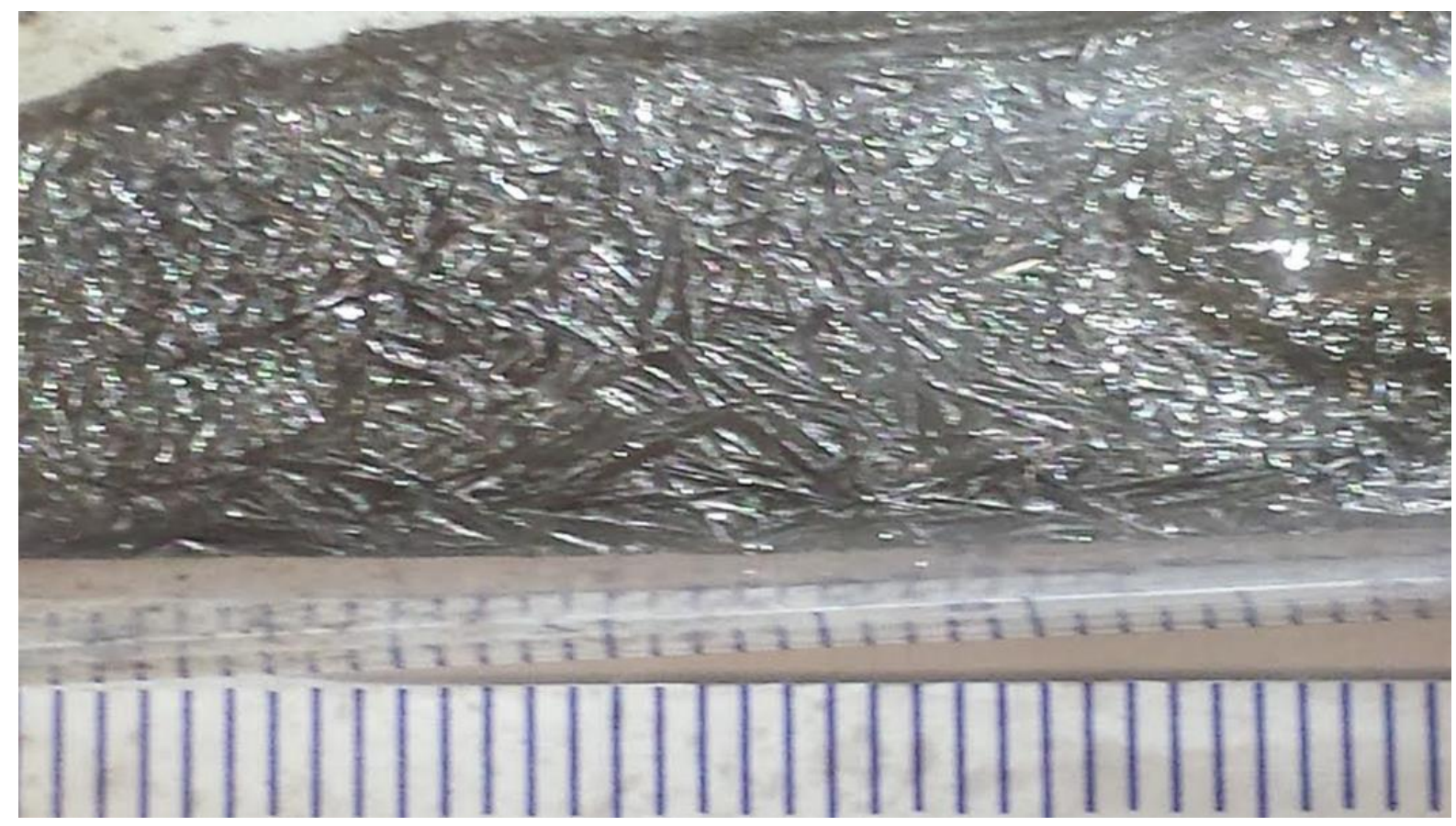

Figure 5. Morphology of nanowires indicating nanocubes as the building block

On the top of the melt in v-l-s conditions, we observed nanocube formation followed by formation of nanowires. However, on the substrate (in this case quartz wall) long nanowires were observed and can be explained by the model where atoms diffuse to whisker which grows in presence of liquid drops which incorporate to form long nanowires. The model results in an expression for the nanowire growth velocity $\mathrm{dL} / \mathrm{dt}$ as a function of the nanowire length $\mathrm{L}$. An integration of this expression yields $\mathrm{L}$ for a given processing time. Johansson et al. use this model to fit nanowire length as a function of nanowire radius data. The model involves three impingement rates (substrate, nanowire sides, and liquid droplet) and two surface diffusion lengths (substrate and nanowires sides). Assuming that all the impingement rates are the same and that the surfaces diffusion lengths are the same greatly reduces the number of parameters. The model is such that one can always pick an impingement rate to obtain the measured nanowire length. The experimental data and theory indicate that the dependence of nanowire length on nanowire radius is complicated and depends on the material and growth conditions.

Alignment of wires in both cases were preferential to both the substrate surface and the NW array as a whole. Since this growth mode is facilitated by controlling only the experimental parameters, the process can be scaled to grow larger NW arrays. Although we did not control the diameter of nanowires in our study, several studies have used catalyst size to control the diameter. From the figure 5 it is clear that coldest location created by quartz tube shows larger length of nanowires compared to solid thallium arsenic selenide material with lower thermal conductivity.

\section{SUMMARY}

The experimental data and theory indicate that the growth mechanism of nanowires is very complex. The dependence of nanowire length on nanowire radius is complicated and depends on the material and growth conditions. Experiments were performed to grow nanowires of lead selenides and thallium arsenic selenide using physical vapor transport and vapor-liquid-solid method. The Experimental data indicated that nanocubes and elongated nanomorphology were building blocks at early stage of nanowire formation when catalyst is not used to initiate growth. However on the quart surfaces long nanowires were observed and can be explained by the model where atoms diffuse to whisker which grows in presence of liquid drops which incorporate to form long 
nanowires. This mechanism is similar to that proposed by Johansson et al. However, further experimental are required to correlate length and growth factors.

\section{ACKNOWLEDGEMENTS}

The supports of Space Life and Physical Sciences Division, Human Exploration and Operations Mission Directorate, NASA Headquarter for student training is gratefully acknowledged.

\section{REFERENCES}

1. N. B Singh, S. R Coriell, Matthew King, Brian Wagner, David Kahler, David Knuteson, Andre Berghman and Sean McLaughlin, Growth Mechanism and Characteristics of Semiconductor Nanowires for Photonic Devices", Journal of Nanoscience and Technology, 1 (2) 1-8 (2014).

2. M. King, N. B. Singh, D. Knueson, A. Berghmans, B Wagner, D. Kahler, " Nanowire-based photodetectors: growth and development of chalcogenide nanostructured detectors, Micro- and Nanotechnology Sensors, Systems, and Applications III", edited by Thomas George, M. Saif Islam, Achyut K. Dutta, Proc. of SPIE Vol. 8031, 803132, (2011.

3. R. L. Parker, R. L. Anderson, S. C. Hardy "Growth and evaporation kinetics and surface diffusion of K and Hg whiskers", Appl. Phys. Lett. 3, 93 (1963).

4. J. Johansson, C. P. Svensson, T. Martensson, L. Samuelson, W. Seifert, "Mass transport model for semiconductor nanowire growth", J. Phys. Chem. B 109, 13567 (2005).

5. V. Schmidt, S. Senz, U. Gosele, "Diameter dependence of the growth velocity of silicon nanowires synthesized via the vapor-liquid-solid mechanism", Phys. Rev. B 75, 045335 (2007).

6. V. G. Dubrovskii, N. V. Sibirev, G. E. Cirlin, J. C. Harmand, V. M. Ustinov, "Theoretical analysis of the vapor-liquid-solid mechanism of nanowire growth during molecular beam epitaxy", Phys. Rev. E 73, 021603 (2006).

7. J. Johansson, B. A. Wacaser, K. A. Dick, W. Seifert, "Growth related aspects of epitaxial nanowires", Nanotechnology 17, S355 (2006). 